\title{
The Radar Echo Telescope for Cosmic Rays: Pathfinder experiment for a next-generation neutrino observatory
}

S. Prohira $\odot,{ }^{1, *}$ K. D. de Vries, ${ }^{2, \dagger}$ P. Allison, ${ }^{1}$ J. Beatty, ${ }^{1}$ D. Besson, ${ }^{3,4}$ A. Connolly, ${ }^{1}$ P. Dasgupta, ${ }^{5}$ C. Deaconu, ${ }^{6}$ S. De Kockere, ${ }^{2}$ D. Frikken, ${ }_{1}$ C. Hast ${ }^{7}$ E. Huesca Santiago, ${ }^{2}$ C.-Y. Kuo, ${ }^{8}$ U. A. Latif, ${ }^{3,2}$ V. Lukic, ${ }^{2}$ T. Meures, ${ }^{9}$ K. Mulrey, ${ }_{10,11}$ J. Nam, ${ }^{8}$ A. Nozdrina, ${ }^{3}$ E. Oberla,${ }^{6}$ J. P. Ralston, ${ }^{3}$ C. Sbrocco, ${ }^{1}$ R. S. Stanley, ${ }^{2}$ J. Torres, ${ }^{1}$ S. Toscano, ${ }^{5}$ D. Van den Broeck, ${ }^{2,10}$ N. van Eijndhoven, ${ }^{2}$ and S. Wissel ${ }^{12,13}$

(Radar Echo Telescope Collaboration)

\author{
${ }^{1}$ Department of Physics, Center for Cosmology and AstroParticle Physics (CCAPP), \\ The Ohio State University, Columbus, Ohio 43210, USA \\ ${ }^{2}$ Vrije Universiteit Brussel, Dienst ELEM, IIHE, Brussels, Belgium \\ ${ }^{3}$ University of Kansas, Lawrence, Kansas 66045, USA \\ ${ }^{4}$ National Research Nuclear University, Moscow Engineering Physics Institute, Moscow, Russia \\ ${ }^{5}$ Université Libre de Bruxelles, Brussels, Belgium \\ ${ }^{6}$ Enrico Fermi Institute, Kavli Institute for Cosmological Physics, Department of Physics, \\ University of Chicago, Chicago, Illinois 60637, USA \\ ${ }^{7}$ SLAC National Accelerator Laboratory, Menlo Park, California 94025, USA \\ ${ }^{8}$ National Taiwan University, Taipei, Taiwan \\ ${ }^{9}$ University of Wisconsin-Madison, Madison, Wisconsin 53706, USA \\ ${ }^{10}$ Vrije Universiteit Brussel, Astrophysical Institute, Brussels, Belgium \\ ${ }^{11}$ Department of Astrophysics/IMAPP, Radboud University, \\ P.O. Box 9010, 6500 GL Nijmegen, The Netherlands \\ ${ }^{12}$ Departments of Physics and Astronomy \& Astrophysics, Institute for Gravitation and the Cosmos, \\ Pennsylvania State University, University Park, Pennsylvania 16802, USA \\ ${ }^{13}$ California Polytechnic State University, San Luis Obispo, California 93407, USA
}

(Received 2 June 2021; accepted 6 October 2021; published 23 November 2021)

The Radar Echo Telescope for Cosmic Rays (RET-CR) is a recently funded experiment designed to detect the englacial cascade of a cosmic ray-initiated air shower via in-ice radar, toward the goal of a fullscale, next-generation experiment to detect ultrahigh energy neutrinos in polar ice. For cosmic rays with a primary energy greater than $10 \mathrm{PeV}$, roughly $10 \%$ of an air shower's energy reaches the surface of a high elevation ice sheet $(\gtrsim 2 \mathrm{~km})$ concentrated into a radius of roughly $10 \mathrm{~cm}$. This penetrating shower core creates an in-ice cascade orders of magnitude more dense than the preceding in-air cascade. This dense cascade can be detected via the radar echo technique, where transmitted radio waves are reflected from the ionization deposit left in the wake of the cascade. RET-CR will test the radar echo method in nature, with the in-ice cascade of a cosmic ray-initiated air shower serving as a test beam. We present the projected event rate and sensitivity based upon a three part simulation using CORSIKA, GEANT4, and Radioscatter. RET-CR expects $\sim 1$ radar echo event per day.

DOI: $10.1103 /$ PhysRevD.104.102006

\section{INTRODUCTION}

Ultrahigh energy cosmic rays (UHECR) and neutrinos (UHEN) are important messengers from the most energetic astrophysical sources. Identifying and understanding these sources are key goals of current multimessenger astronomy,

prohira.1@osu.edu

'Krijn.de.Vries@vub.be a burgeoning field with exciting recent breakthroughs and many discoveries to be made [1-3].

The primary challenge to detecting UHECR and particularly UHEN is the low flux at the highest energies. This low flux requires an observatory that can efficiently probe a large target volume, in order to acquire a statistically significant sample of events. There are several current and proposed experimental strategies to achieve this large volume [4-10]. In this paper, we discuss the radar echo method. This method has promising projected sensitivity to 


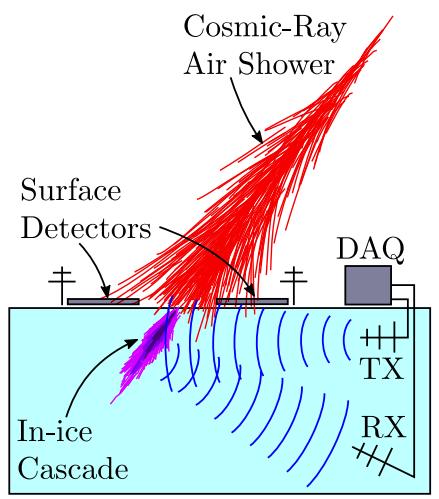

FIG. 1. The RET-CR concept. A surface detector triggers on the charged in-air cascade particles as they reach the surface. The energy of this in-air cascade is deposited into the ice, where a denser in-ice cascade is produced. Radio waves are broadcast from the transmitter (TX) and reflected from the in-ice cascade to the embedded receiver ( $\mathrm{RX})$.

neutrinos in the $10-100 \mathrm{PeV}$ range, providing complementarity with existing and future techniques for measuring UHEN [11-16].

A high energy particle (e.g., neutrino) interacting in a dense medium (e.g., ice) creates a cascade of relativistic particles that ionize atoms in the target medium. A shortlived cloud of charge is left behind, which can, if sufficiently dense, reflect incident radio waves. RET proposes to illuminate a volume of ice with a transmitter radio-frequency antenna and monitor that same volume for reflections with a receiving antenna. To improve reconstruction of the geometry of a cascade, and therefore the progenitor source direction, a target volume can be illuminated with multiple transmitters and monitored with multiple receivers. Overall, the radar echo method allows for the coverage of a large volume with minimal apparatus and station layout optimized for a given neutrino energy, making it an attractive option for UHEN detection.

The radar echo method has been verified in the lab [11,12], with first observations of radar echoes from particle cascades recently reported $[15,16]$. These lab tests are critical steps in developing an ultimate radar echo neutrino observatory. The final step in validating the technique is to translate the laboratory tests into nature, and test the method in situ with a known test beam: the inice cascade produced when the extensive air shower (EAS) of an UHECR impacts the ice.

In this paper, we describe the Radar Echo Telescope for Cosmic Rays (RET-CR) (Fig. 1) which will serve as a test bed for the radar echo method and a final stepping stone toward the eventual construction of a full-scale radar echo telescope for UHEN.

\section{HISTORY, THEORY, AND BACKGROUND}

First efforts on the radar echo method were chronicled by Lovell [17]. With collaborator Blackett, the Jodrell bank observatory was constructed in the United Kingdom, anticipating that radar echoes from UHECR might explain "sporadic radio reflexions" from the upper atmosphere. Ultimately, those signals were determined to be from meteors, which ionize similar, far denser trails in the upper atmosphere. After several experimental efforts, including the Telescope Array RAdar (TARA) experiment, failed to detect UHECR via radar [18-21], and theoretical work explaining the lack of observed reflections [22,23], the inair method was finally deemed not viable due to short ionization lifetimes in the atmosphere at EAS altitudes and damping from collisions between ionized electrons and neutral air molecules (an issue first raised by Eckersley in 1941 [17], though largely subsequently ignored.)

Neutrino interactions in the ice produce ionization densities many orders of magnitude more dense than those in air, owing to the $\sim 10^{3}$ greater density of ice relative to air. Therefore, while the ionization lifetime remains short in ice (roughly $10 \mathrm{~ns}$ [24]) and the collision rate is extremely high, so too is the underlying ionization density, allowing for a possible scatter. Several models now exist for the in-ice radar echo $[13,14]$ and show promising experimental sensitivity. Laboratory tests have shown good agreement with theory, but in order to test the radar echo method in nature, a source of in-ice ionization is required; EAS offers such a source.

The EAS of a UHECR expands radially outward from the shower axis, such that an EeV cascade has a $\mathcal{O}(100 \mathrm{~m})$ footprint on the ground. However, nearly all of the cascade energy is contained within $\sim 10 \mathrm{~cm}$ of the shower axis, as illustrated below [25]. The sought-after signal depends on the total number of particles in the shower core, as well as the geometry of the shower. In Fig. 2, we plot the ratio of

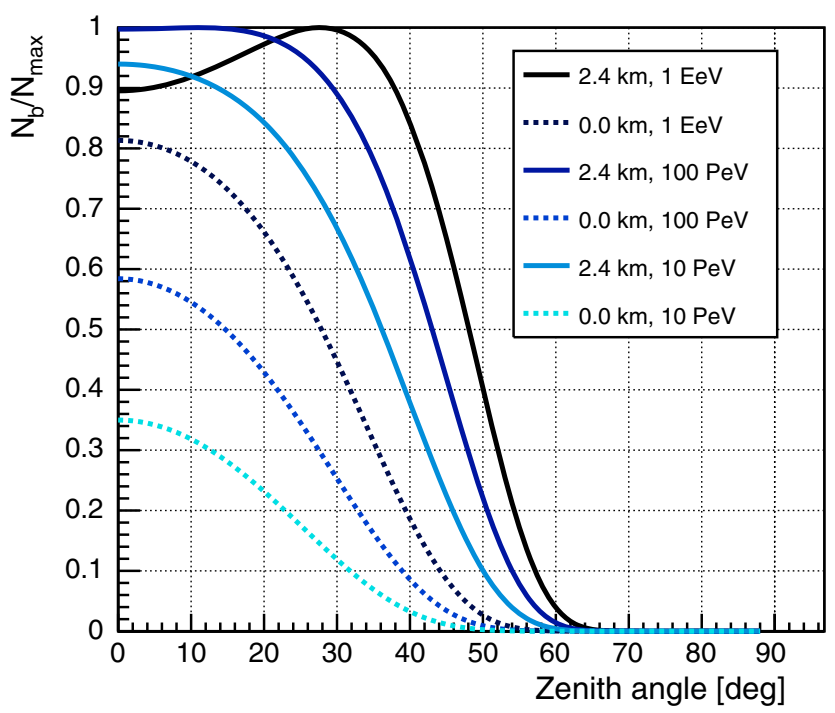

FIG. 2. The ratio of the number of cascade particles, $\mathbf{N}_{b}$, arriving at the air/ground boundary relative to the maximum number in the cascade, $\mathrm{N}_{\max }$, for various energies and ground elevations. At a $2.4 \mathrm{~km}$ elevation and $1 \mathrm{EeV}$ primary energy, shower maximum at normal incidence is in ice. 
the number of particles at the air/ground boundary $\left(\mathrm{N}_{b}\right)$ to the maximum number in the cascade $\left(\mathrm{N}_{\max }\right)$ for different primary UHECR energies for different ice elevations as a function of zenith angle using the Nishimura-KamataGreisen (NKG) approximation [26,27]. For a high elevation (such as the interior Antarctic plateau), this ratio is $\geq 0.9$ for energies $\geq 10 \mathrm{PeV}$ for a wide zenith angle range, indicating that a significant fraction of the energy of the primary particle will arrive at the air/ice boundary.

The core of a UHECR when it impacts the ice can be used as an in-nature test beam. Though TARA demonstrated that UHECR detection in air via radar echo was not feasible, the in-ice cascade produced by the remaining EAS particles, as demonstrated by our beam tests, should be detectable via radar echo (for discussion of how the in-ice casade may be detected by Askaryan-type detectors, see Refs. [28,29]). For energies above $10 \mathrm{PeV}$, we expect an ionized column with a density that decreases rapidly with radius and an in-ice length of about $10 \mathrm{~m}$. The effective radius along this column at which radar will scatter depends on the transmitter frequency (discussed below); for frequencies in the $100 \mathrm{~s}$ of $\mathrm{MHz}$ range, this radius is approximately $10 \mathrm{~cm}$. The profile of this ionization deposit is similar to that which would be produced by a neutrinoinduced cascade, the primary difference being that neutrino events are more likely to occur in deep ice rather than near the surface.

The properties of the in-ice cascade from EAS have been studied using CORSIKA [30] to simulate the extensive air shower evolution, GEANT-4 [31] to simulate the propagation of these cascades once they enter the ice and RadioScatter [32] to calculate the reflected signals from the ionization deposits left in the wake of the cascades. We next discuss our planned detector layout and design and then describe our simulation and projected sensitivity.

\section{EXPERIMENTAL CONCEPT}

The experimental concept is shown in Fig. 1. A transmitter illuminates the region of ice just below the surface, with receivers monitoring this same region. The EAS of a UHECR with a primary energy greater than $10^{16} \mathrm{eV}$ deposits a fraction ( $\gtrsim 10 \%)$ of the primary energy at the surface of a high-elevation ice sheet. This energy is largely centered around the cascade axis, resulting in a dense secondary in-ice cascade roughly $10 \mathrm{~m}$ long. The charged particles from the EAS are detected by a surface scintillator array, triggering a radio receiver waveform to be recorded in the radar data acquisition system (DAQ). This simple setup closely parallels that already employed for the laboratory test-beam experiment, with the focus of the experiment on post-run, offline analysis of the data. This relative simplicity also allows for testing various radarbased trigger routines, which can be evaluated against the charged particle trigger. Such testing is critical, as an

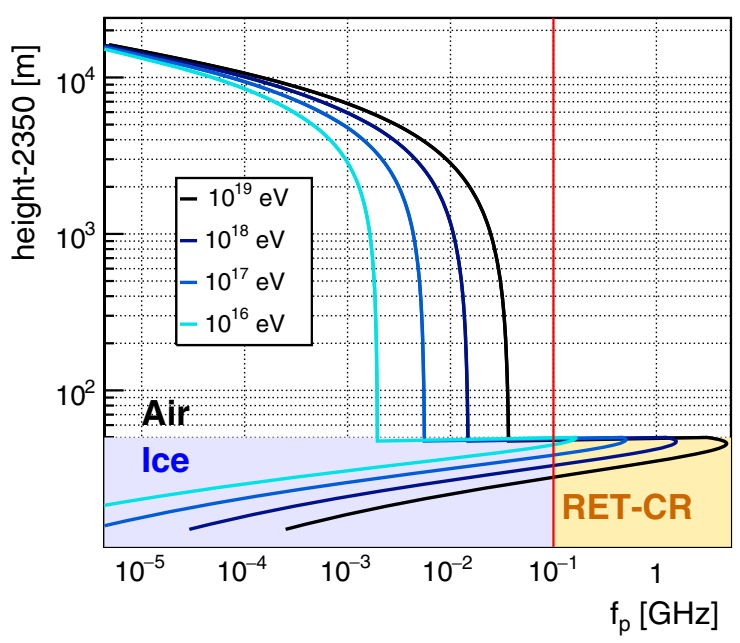

FIG. 3. The peak plasma frequency $\left(f_{p}=\omega_{p} / 2 \pi\right)$ of the cascade as it develops in air and then in ice, at normal incidence. The in-ice cascade is far more dense than the in-air cascade, making it detectable via radar for primary energies greater than $10^{16} \mathrm{eV}$. The red line indicates the point at which $f_{p}>100 \mathrm{MHz}$.

eventual neutrino detector will be triggered by the radar signal itself.

When the cascade leaves the air and enters the ice, the density of the resultant ionization increases dramatically. This results in an ionization deposit in the ice with a plasma frequency $\omega_{p}=\sqrt{4 \pi n_{e} q^{2} / m}$ far higher than any point in air. The plasma frequency, with $n_{e}$ the number density of the ionization, $q$ the electric charge, and $m$ the electron mass, is a measure of the density of an ionization deposit. To first order, incident fields with interrogating frequencies lower than $f_{p}=\omega_{p} / 2 \pi$ are reflected efficiently. ${ }^{1}$ The profile of $f_{p}$ from a primary cosmic ray as it moves through air into the ice is shown in Fig. 3, where the in-air and in-ice components of an EAS are indicated, as well as a vertical line indicating $100 \mathrm{MHz}$. For the in-ice portion of EAS with primary energies greater than $10^{16} \mathrm{eV}$, $f_{p} \gtrsim 100 \mathrm{MHz}$, indicates efficient scattering for interrogating frequencies in this range.

In the following sections, we describe the various subsystems of RET-CR. We provide details of the experimental layout in Appendix A.

\section{A. Surface detector}

The RET-CR surface detector is designed to detect the air shower incident on the surface of the radar detector, providing both an external trigger to the radar DAQ and

\footnotetext{
${ }^{1}$ In a collisionless plasma, $\omega_{p}$ is a hard cutoff between the "overdense" and "underdense" regimes, which indicate fully opaque (reflective) or semitransparent plasma, respectively. When taking collisions into account (as we do in our simulations), this boundary is smeared, but $\omega_{p}$ is still a useful discriminator for the underlying ionization density.
} 
an independent reconstruction of the air shower. Using the surface detector as a trigger for the radar system will ensure that an UHECR has entered the radar detector volume with sufficient energy to be detected through the radar echo technique. The independent reconstruction of shower parameters by the surface detector will provide values for the core position, energy, and arrival direction of the incident UHECR. These values will then be used to validate the reconstruction parameters obtained by the radar echo system.

The primary component of the surface detector is a scintillator plate array. The plates will be grouped in pairs following the design of the Cosmic-Ray Energy CrossCalibration Array [33,34]. The plates in each pair of scintillators will be separated by $20 \mathrm{~m}$. The scintillators will be accompanied by a butterfly radio antenna operating in the frequency band 30-300 MHz to form a station. Each station will have its own DAQ and power system. The combination of a radio and scintillator signal at each station will be beneficial in providing event reconstruction and energy estimates (more details below). The current deployment layout is shown in Appendix A, where the stations are grouped into two sets of three stations, separated by the central radar system. Additionally, a system diagram is provided in Appendix B. The station layout has been optimized for trigger efficiency, discussed below.

The current prototyping and simulation development work utilizes the scintillators from the KASCADE experiment [35]. The butterfly radio antennas have been donated by the CODALEMA/EXTASIS experiment [36,37]. As such, for modeling the polyvinyl-toluene scintillator in GEANT4, we use a carbon:hydrogen ratio of 9:10 [38]. The panels deployed to the field will be similar in size and composition, and we do not expect any difference in performance from the panels simulated here.

The scintillator trigger threshold is tuned to maximize the radar echo detections. Simulations indicate that air showers with energy less than $10^{15.5} \mathrm{eV}$ are inadequate to produce an in-ice cascade detectable via radar echo (and the rate of such showers would overwhelm the DAQ storage and may cause interesting events at higher energies to be lost). Additionally, simulations of the air shower radio footprint show that radio reconstruction of the air shower is not possible for showers with an energy less than approximately $10^{16.0} \mathrm{eV}$. Therefore, we target $100 \%$ efficiency at $10^{16.5} \mathrm{eV}$, with efficiency defined as the percentage of cosmic ray air showers traversing the instrumented area that trigger the surface detector. We aim for a trigger rate of order $10^{5}$ events per month, leading to approximately 300 surface triggered events a day. This is a manageable rate for both the surface and radar DAQ systems.

Simulation studies have been conducted to determine an appropriate triggering scheme for the surface detector. Events have been simulated with energies in the range from $10^{15.0}$ to $10^{19.0} \mathrm{eV}$ and zenith angles in the range of $0-30^{\circ}$. We limit ourselves to this zenith range because inice energy deposition decreases dramatically beyond a $30^{\circ}$ zenith. At higher energies, cascades at zenith angles $>30^{\circ}$ will likely be detectable via radar and will increase our event rate slightly relative to what we present here (e.g., for $\mathrm{EeV}$ cascades, it could increase the rate by up to a factor of 2). The simulations were made using the CORSIKA and CoREAS [39] software for air shower simulation with a ground elevation set to $2400 \mathrm{~m}$, that of an optimal deployment site, Taylor Dome, Antarctica. A change in this altitude will affect the point within the shower development at which the air shower passes through the detector. Showers at sea level are generally developed beyond shower maximum before reaching the ground. At the altitude of Taylor Dome, whether the shower has developed to a point before or after the shower maximum is strongly dependent on the energy and zenith angle of the air shower (Fig. 2).

The stations of the surface detector will trigger independently. Each scintillator will be required to contain a deposit of $6 \mathrm{MeV}$ (1 minimally ionizing particle) or greater per event, and both scintillators in a station must trigger coincidentally within an event (an L0 trigger). The final trigger requirement is that all stations within one cluster must have coincident, above threshold energy deposits in all scintillators (an L1 trigger). The width of the time window for this coincidence, $\sim 170 \mathrm{~ns}$, corresponds to the maximum time-difference-of-arrival between two stations separated by roughly $100 \mathrm{~m}$ for a $30^{\circ}$ zenith angle cascade. The resulting trigger efficiency is shown in Fig. 4. In this figure, we show that we achieve $100 \%$ efficiency at $10^{16.5} \mathrm{eV}$, as desired. Decreasing a half-decade in energy, at $10^{16.0} \mathrm{eV}$ the efficiency decreases to $\sim 70 \%$. In the lowest energy bin simulated, the efficiency is approximately $5 \%$. This rapid turn-on in our trigger threshold allows us to target our desired event rate.

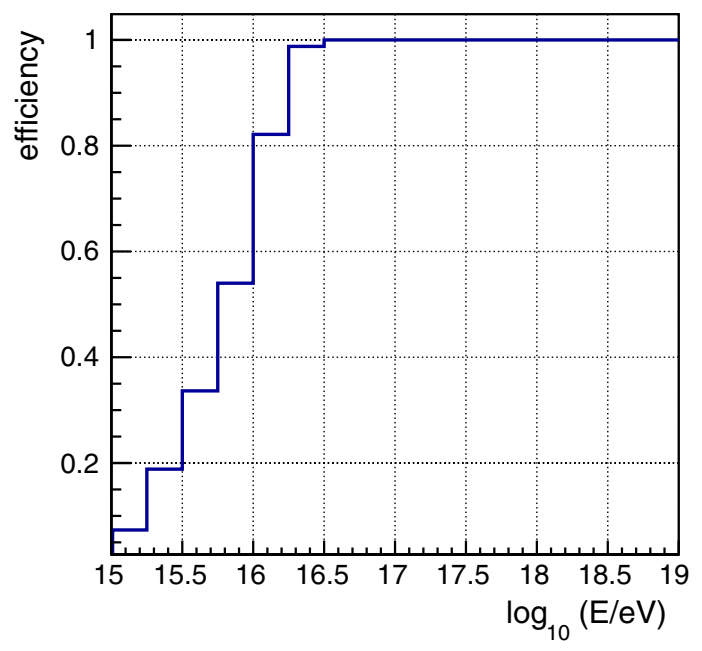

FIG. 4. Trigger efficiency curve for the surface system as a function of primary UHECR energy. 
The radio component of the surface system operates at $1 \mathrm{GS} / \mathrm{s}$, allowing for precise angular reconstruction of cascades, which can then be used to test the reconstruction capabilities of the radar detector. Similar radio-based cosmic ray detectors with comparable baselines can reconstruct arrival direction with an error less than 1-2 ${ }^{\circ}$ [40-43]. At the time of writing, a set of three surface stations are taking data in a rooftop test configuration with $\sim 100 \mathrm{~m}$ baselines. Analysis of these data will provide an accurate number on angular reconstruction of the surface system in advance of RET-CR.

Furthermore, the radio component of the surface system also provides a measurement of the energy of the cascade. Similar radio-based cosmic ray detection experiments $[34,41,44]$ including those performing reconstruction with a limited number of antennas [45] can constrain energy to approximately $15 \%-20 \%$, with some studies [46] claiming $15 \%$ resolution with just a single station. The RET-CR surface stations will have a slightly wider bandwidth than many of these experiments, so we expect a similar-if not slightly better-energy uncertainty. Our rooftop test data will provide an accurate number for the energy uncertainty of our specific system in advance of RET-CR.

To estimate the core reconstruction accuracy, we developed a reconstruction procedure using the realistic particle deposit simulated with CORISIKA. For a given simulation, different core positions were chosen, and the scintillator deposit was determined, conservatively assuming a $15 \%$ uncertainty in measured deposit. We then used a minimization technique based on the NKG function to reconstruct the core position, similar to what is done in Ref. [35]. Using this method, we were able to reconstruct the core with a mode (68\% quantile) resolution of 7.8 (24.4) $\mathrm{m}$ at $10^{1} 6 \mathrm{eV}$ and 10.8 (24.6) $\mathrm{m}$ at $10^{1} 7 \mathrm{eV}$ for showers with a zenith angle less than $30^{\circ}$. This is an upper bound on the core resolution, as we develop more advanced core fitting methods making use of radio measurements, which should improve reconstruction for the outlier events that inflate the size of the $68 \%$ interval.

\section{B. Radar echo detector}

\section{Data acquisition system}

The primary element of the radar echo detector DAQ is a Xilinx RFSoC [47]. This all-in-one device will be used for both the transmitter and receiver components. The transmit portion comprises eight channels, each with a $6 \mathrm{GS} / \mathrm{s}$ digital-to-analog converter (DAC) capable of producing a phased, modulated output to an array of transmitters. The receive portion has eight channels, each channel with a $4 \mathrm{GS} / \mathrm{s}$ analog-to-digital converter. A Virtex-7 FPGA (field-programmable gate array) provides transmit and receive functions and trigger logic, and an on-board ARM processor facilitates information transfer between the FPGA and the communication subsystems, also described below.
The DAQ will have local storage for buffering data and a prioritizer system for telemetry, with a design expectation that our data transfer rate will be the primary bottleneck in getting data from the station. The most promising events are sent via a communication link as well as being stored locally on disk. The criteria for this can include (i) measured primary energy from the surface detectors or (ii) the proximity of the in-ice vertex to a receive antenna or a combination of the two. The disks will be retrieved at the end of the season.

The FPGA will host a number of radar "triggers" with potentially varying topologies, even though these triggers do not actually signal an event snapshot. These triggers will be trained against the surface detector trigger to determine their efficacy for eventual use in the successor neutrino detector, where the radar signal itself must trigger the DAQ. One such trigger under investigation is a heterodyne trigger (also called a "chirp trigger") based upon a method developed for the TARA remote stations $[48,49]$ that exploits the frequency shift of the return signal. The geometry of RET-CR is such that all of the received radar echoes will exhibit this frequency shifting behavior. Other triggers based on the unique radar signature are also being explored. The sensitivity studies in this article employ a simple threshold trigger for the radar component.

\section{Transmit array and transmitter modulation}

The transmitter for RET-CR will be a vertical phased array of eight vertically polarized antennas buried 2-20 m below the ice surface. The exact depth requires further study of ice properties and a better understanding of radio wave propagation near the surface of the ice through ongoing simulations, as discussed in Sec. IV C. This phased array serves two critical functions. The first is directionality - a phased array governed by an FPGA can form high gain beams in a defined direction, achieved via adjusting the relative phases of the transmit signal being delivered to each of the antennas in the array. A vertical phased array has azimuthal symmetry with a high gain beam at a defined zenith angle, defined by the relative phase delays of each antenna. Since our reflectors are confined to the top $\sim 10 \mathrm{~m}$ of the ice just below the surface, we can steer the beam slightly upward virtually no power is beamed to the region below, where we do not expect to receive UHECR core reflections. Recent studies in Antarctica have shown that in-ice phased arrays are highly efficient receivers [50], and phased transmitter arrays are common in use throughout the world, including the TARA experiment. The second critical function of a phased array is to lower the single-amplifier gain for the transmit power amplifier. In lieu of a single $160 \mathrm{~W}$ power amplifier, each antenna will have its own $20 \mathrm{~W}$ power amplifier. This distributes the ohmic heating losses over eight antennas instead of one and provides some redundancy; in the event 
that a power amplifier malfunctions, the experiment loses some efficiency but does not shut down entirely.

The antennas will be based upon the simple bicones or biconical dipoles used by the RICE, ARA, and ARIANNA experiments in Antarctica. These antennas are small enough to fit down a borehole but are sufficiently broadband as to allow for a range of transmit frequencies and modulations. Simulations using finite-difference timedomain [51] and parabolic equation codes [52,53] are underway to determine if nonuniform antenna spacing or antennas with asymmetric zenith angle gain can increase transmitter efficiency in the direction the beam is "steered". Some recent studies also indicate that broadband phased arrays may be possible in ice [54], though focus here is on higher frequencies than those of interest to RET-CR.

The modulation scheme is currently being defined. We plan to frequency hop or frequency shift around a central carrier of 100-300 MHz, with a transmitter bandwidth of 50-100 MHz. The central frequency is determined by maximizing the signal to noise ratio of a radar signal to the background noise. The signal has an optimal frequency dependent on the cascade dimensions and density, and the noise decreases with increasing frequency as thermal noise begins to dominate over galactic noise above $\sim 150 \mathrm{MHz}$.

This central frequency and the ultimate modulation strategy will be determined via simulations that are already underway. Modulation, as opposed to pulsing, increases detector live time, as long as the carrier signal can be removed from the receivers. We discuss this below in Sec. III B 4.

\section{Receive array}

The receiver array will be laid out in the configuration shown in Appendix A. Two different TX-RX baselines allow for a wide range of primary particle energies to be detected. Similar to the transmitter array, the receiver antennas will be buried 2-20 m below the surface of the ice. Each receive antenna will be a vertical phased array, similar to the transmit array, in order to maximize nearsurface gain with full azimuthal coverage.

The receivers will not trigger the DAQ but will form triggers as a test bed for eventual use in a neutrino array.

\section{Amplification and adaptive filtration}

We will have a limiter and high-gain, low-noise amplifier on each receive channel, providing protection during transmitter turn-on and approximately $70 \mathrm{~dB}$ of gain, respectively. This amount of gain is sufficient to attain the galactic noise floor at our frequency, location, and receiver bandwidth of $\sim 100 \mathrm{MHz}$.

Because radar receivers will be illuminated by the transmitter, it is essential to filter the transmitter or gate the receivers such that amplifier saturation does not occur.
We plan to adopt an adaptive filtration scheme, whereby we will record an amplifier-bypassed snapshot of the transmitted signal over a horizon-distance window in time at each receiver and then inject it time delayed and phase inverted into the receiver stream before the amplifier chain. The delay and output amplitude are tuneable, allowing for an iterative reduction of the input amplitude until the carrier is fully eliminated. This procedure will be updated at intervals throughout the day to account for environmental changes such as snow accumulation, which have been shown to introduce measurable changes in reflection times on $\sim$ day timescales [55].

\section{Power, system health, calibration, and communications}

The detector will be fully autonomous and powered by three $1.2 \mathrm{~kW}$ solar arrays arranged in a triangle, such that at any time of (a sunny) day the station is provided with approximately $1 \mathrm{~kW}$ of power, with the majority of this power being used by the transmit power amplifiers. A bank of batteries will buffer power to assist in running the stations during adverse weather conditions. Each surface station will be powered by an individual photovoltaic. RET-CR will run only during the austral summer.

The system health, including power consumption, DAQ enclosure temperature, power amplification health, and local weather will all be monitored remotely, in real time.

We will deploy a small, autonomous calibration unit that sends out a broadband pulse at regular intervals. This unit will serve as a regular baseline for thresholds and ensure global time synchronization, as well as for active monitoring of the above mentioned environmental changes, such as snow accumulation.

The communication system will be a two-way satellitebased Internet link. Through this link we will telemeter the prioritized data and system health information back to the lab and, from the Northern Hemisphere, new trigger schemes and other station software and commanding to RET-CR. Alternative communications links via point-topoint Ethernet may be possible if there are line-of-sight repeater stations between a major base and the remote RET-CR.

\section{PROJECTED SENSITIVITY}

The approximate sensitive surface area instrumented by RET-CR is $5 \times 10^{4} \mathrm{~m}^{2}$. Through this area we can expect a flux of roughly 1 event at $100 \mathrm{PeV}$ per day. The surface system will trigger on every cosmic ray with a primary energy above this, with decreasing efficiency at lower energies, as described in Sec. III A. To simulate our radar echo detection efficiency, we performed a detailed multistep Monte Carlo simulation which we describe here using (in order) CORSIKA, GEANT4, and RadioScatter. 


\section{A. CORSIKA cascades and the surface stations}

A CORSIKA-based Monte Carlo simulation for optimizing the surface array location was described in Sec. III A. This same distribution of events (core positions, zenith angles, and energies) was used to simulate the radar sensitivity of RETCR. A separate set of CORSIKA simulations was prepared specifically for producing the GEANT4 output used in subsequent simulation steps. These CORSIKA showers were produced at 0,15 , and $30^{\circ}$ zenith for each half-decade energy, with a ground elevation of $2400 \mathrm{~m}$, as before. For $10^{16}$ and $10^{16.5} \mathrm{eV}$, no thinning was employed. For higher energies, thinning is set to $10^{-7}$ of the primary particle energy. For $10^{17}$ and $10^{17.5} \mathrm{eV}$, the CORSIKA "weight" of a single particle will never be larger than 10 ; for $10^{18} \mathrm{eV}$, it will never be larger than 100 . Thinning retains the overall energy

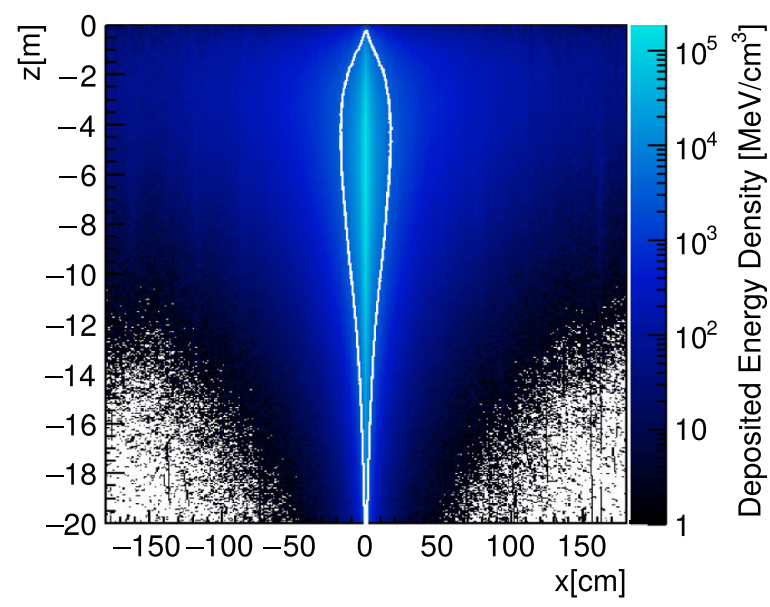

FIG. 5. A one centimeter wide, two-dimensional slice of the inice energy density distribution along the cascade axis for a primary proton with $\mathrm{E}=10^{17} \mathrm{eV}$. The solid white line outlines the region for which the plasma frequency exceeds $100 \mathrm{MHz}$.

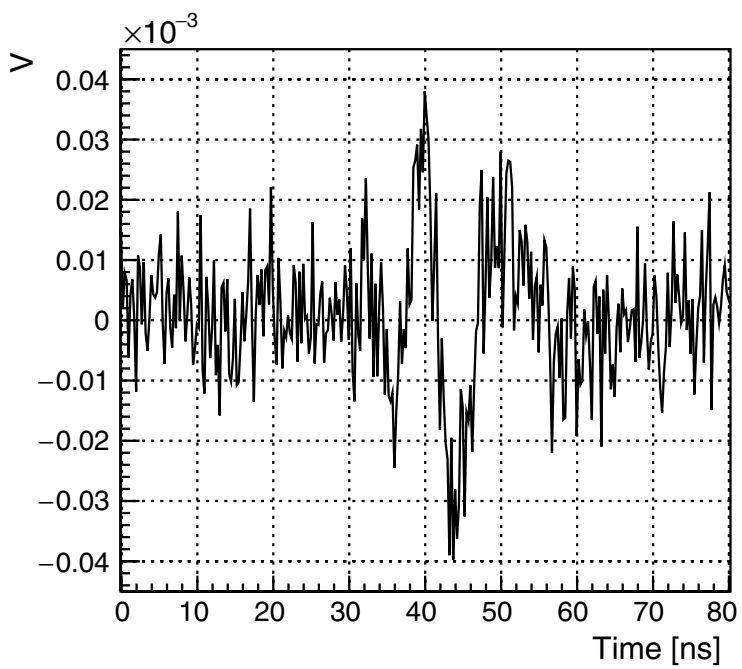

of a cascade, such that the total in-ice ionization number will be the same for any thinning, but it changes the distribution of low-energy particles in the final footprint (which is then used as the input to GEANT4). We therefore minimized thinning as much as possible, subject to computing constraints.

\section{B. GEANT4 simulations from CORSIKA output}

The CORSIKA particle output at the surface of the ice was subsequently used as input for the GEANT4 simulation code configured to propagate particles into the ice. For this, a realistic density profile similar to that found at South Pole was used, $\rho(z)=0.460+0.468 \times\left(1-e^{-0.02 z}\right)$ with $\rho$ the density in $\mathrm{g} / \mathrm{cm}^{3}$ and $z$ the depth in $\mathrm{m}$. In each step of the simulation, the ionization energy loss is recorded; taking a typical ionization energy of $50 \mathrm{eV}$ allowed us to obtain the free charge density profile in the ice [56]. An example of this profile is shown in Fig. 5 for an air shower induced by a $10^{17} \mathrm{eV}$ proton primary incident on an ice sheet at $2400 \mathrm{~m}$ elevation. The plasma frequency is a good indicator of the reflective properties of the induced plasma, as discussed previously. The solid white line in Fig. 5 outlines the region for which the plasma frequency has a value larger than $100 \mathrm{MHz}$, where fully coherent scattering is expected.

\section{RadioScatter simulations of GEANT4 output}

To simulate the overall sensitivity, we use the Radioscatter [32] code. Radioscatter is a particle-level $\mathrm{C}++$ code to simulate radio scattering from ionization deposits. It calculates the received radio signal reflected from an ionization deposit (from, e.g., a particle cascade) for an arbitrary geometry of transmitter(s) and receiver(s).

The energy deposition calculated by GEANT4 was used as the input to RadioScatter. An example of a triggered event from a $10^{16.5} \mathrm{eV}$ primary at normal incidence is shown in Fig. 6. Clearly visible is the characteristic frequency shift

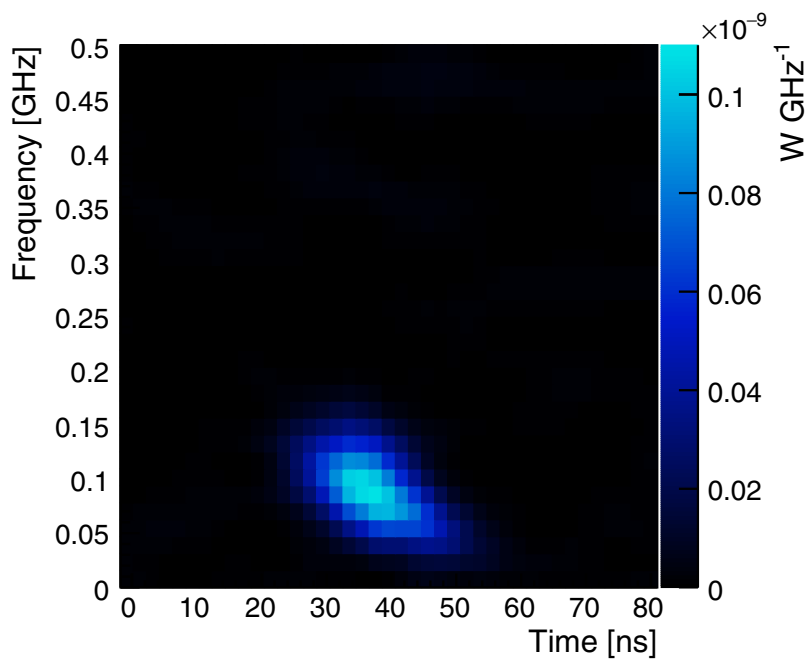

FIG. 6. An example event simulated using CORSIKA+GEANT4+RadioScatter, as described in the text. This is a $10^{16.5} \mathrm{eV}$ primary at normal incidence with a $10 \mathrm{~ns}$ plasma lifetime, simulated with a $160 \mathrm{~W}$ transmitter at $100 \mathrm{MHz}$. 
expected for the RET-CR geometry, which can be exploited in trigger routines. At each cascade position in the surface scintillator simulation set, we simulated two different GEANT4 cascades: (1) the cascade with the closest halfdecade energy below the primary energy of the surface simulation and (2) the cascade with the closest half-decade energy above the primary energy of the surface simulation. This is done because generating the GEANT4 cascades from the CORSIKA output is computationally expensive, so each discrete energy and zenith angle cannot be simulated individually. This method bounds the amount of energy that could arrive at the surface and accounts for shower-toshower fluctuations. Both energies were simulated at each cascade position at one of three zenith angles, 0,15 , and $30^{\circ}$ selected according to their proximity to the "true" zenith angle of the cascade from the surface simulation. These cascades were generated with a uniform distribution in $\cos \theta$ because cosmic rays arrive isotropically at Earth, and a uniform distribution in $\cos \theta$ ensures that any zenith angle dependencies of the trigger are reflected in the sensitivity. The horizon distance for an in-ice transmitter is finite owing to the changing index of refraction in the firn (the top $\sim 100 \mathrm{~m}$ of an ice sheet where snow is being compacted into ice) [57-60]. We therefore put a hard cut on a horizon distance of $150 \mathrm{~m}$, which is commensurate with the point at which the in-ice shower maximum is out of view for a transmitter depth of $\sim 20 \mathrm{~m}$. The simulations in this paper eschew the typical ray-tracing approach for studying propagation in the firn since this has recently been shown to be incomplete without in situ studies of the ice density profile [52,61]. The hard horizon cutoff for the results presented here are a proxy for the loss in efficiency due to propagation effects. These effects will be explored in detail in a future work.

\section{Calculation of the event rate for RET-CR}

The two components of the event rate are the effective area of the detector and the cosmic ray flux. We define both of these over an energy bin $E_{i}$ with index $i$ and width $d E$. The effective area $\mathcal{A}_{i}^{\text {eff }}\left(E_{i}\right)\left[\mathrm{m}^{2}\right]$ for energy bin $i$ is a function of the cross-sectional area $\mathcal{A}_{\perp}$ over which the sensitivity is calculated and a dimensionless trigger efficiency for the same bin, $\mathcal{T}_{i}\left(E_{i}\right)$. To detail the effective area, we first introduce the boxcar function, $\mathbf{B}$, which bounds a number $x_{1}<x<x_{2}$,

$$
\mathbf{B}\left(x, x_{1}, x_{2}\right)=\Theta\left(x-x_{1}\right) \Theta\left(x_{2}-x\right),
$$

where $\Theta$ is the Heaviside step function. Then we define our trigger conditions $\delta^{\mathbf{S}}$ (for surface) and $\delta^{\mathbf{R}}$ (for radar) which are 1 if the trigger condition is satisfied, and 0 if not. For example,

$$
\delta^{\mathbf{R}}=\Theta\left(v^{\text {peak }}-v^{\text {thresh }}\right)
$$

for a peak waveform voltage $v^{\text {peak }}$ and threshold voltage $v^{\text {thresh }}$, and $\delta^{\mathbf{S}}$ is 1 when the surface system trigger logic is satisfied (a coincidence between surface stations with a certain per-station energy threshold, as described in Sec. III A). We then define the number of detected events, for event index $k$, energy bin with index $i$, and $\theta$ bin with index $j$, as a matrix $n_{i j}$. These events are weighted by $\cos \theta_{k}$ to account for the correction to the perpendicular crosssectional area $\mathcal{A}_{\perp}$ seen by a cosmic ray at zenith angles greater than zero,

$n_{i j}\left(E_{i}, \theta_{j}\right)=\sum_{k} \delta_{k}^{\mathbf{S}} \delta_{k}^{\mathbf{R}} \cos \left(\theta_{k}\right) \mathbf{B}\left(E_{k}, E_{i}, E_{i+1}\right) \mathbf{B}\left(\theta_{k}, \theta_{j}, \theta_{j+1}\right)$,

where $n_{i j}$ will be zero for bins in zenith outside of the aperture of the instrument. For RET-CR, this aperture is $0-30^{\circ}$ as discussed in Sec. III A. The total number of simulated events $N_{i j}$, also as a matrix in $E$ and $\theta$ is

$$
N_{i j}\left(E_{i}, \theta_{j}\right)=\sum_{k} \mathbf{B}\left(E_{k}, E_{i}, E_{i+1}\right) \mathbf{B}\left(\theta_{k}, \theta_{j}, \theta_{j+1}\right) .
$$

The trigger efficiency $\mathcal{T}_{i j}$ in energy and zenith bins is represented as the ratio of these two,

$$
\mathcal{T}_{i j}\left(E_{i}, \theta_{j}\right)=\frac{n_{i j}}{N_{i j}},
$$

and we can then sum over all $\theta$ to get this expression as a function of energy only,

$$
\mathcal{T}_{i}\left(E_{i}\right)=\sum_{j} \frac{n_{i j}}{N_{i j}},
$$

meaning that the effective area for energy bin $E_{i}$ is

$$
\mathcal{A}_{i}^{\text {eff }}\left(E_{i}\right)=\mathcal{T}_{i} \mathcal{A}_{\perp} .
$$

The flux as a function of energy $\mathcal{F}(E)\left[\mathrm{m}^{-2} \mathrm{~s}^{-1} s r^{-1} \mathrm{eV}^{-1}\right]$ is a broken power law fit to the measured CR flux by many experiments $[56,62,63]$. To get a number of events per square meter, per second, per steradian, in energy bin $E_{i}$, we integrate $\mathcal{F}(E)$ over the energy range of bin $i$,

$$
\mathcal{F}_{i}\left(E_{i}\right)=\int_{E_{i}}^{E_{i+1}} \mathcal{F}(E) d E .
$$

Finally, the expression we use to calculate the event rate as a function of energy, for energy bin index $i, \mathcal{R}_{i}\left(E_{i}\right)$, is given in Eq. (9),

$$
\mathcal{R}_{i}\left(E_{i}\right)=\mathcal{A}_{i}^{\text {eff }} \mathcal{F}_{i} \int d t \int d \Omega,
$$

where the integral over time is the detector live time, and $\int d \Omega=\int d \phi d(\cos \theta)=\int d \phi \sin \theta d \theta$ is the integral over the aperture of the instrument. For an experiment sensitive 

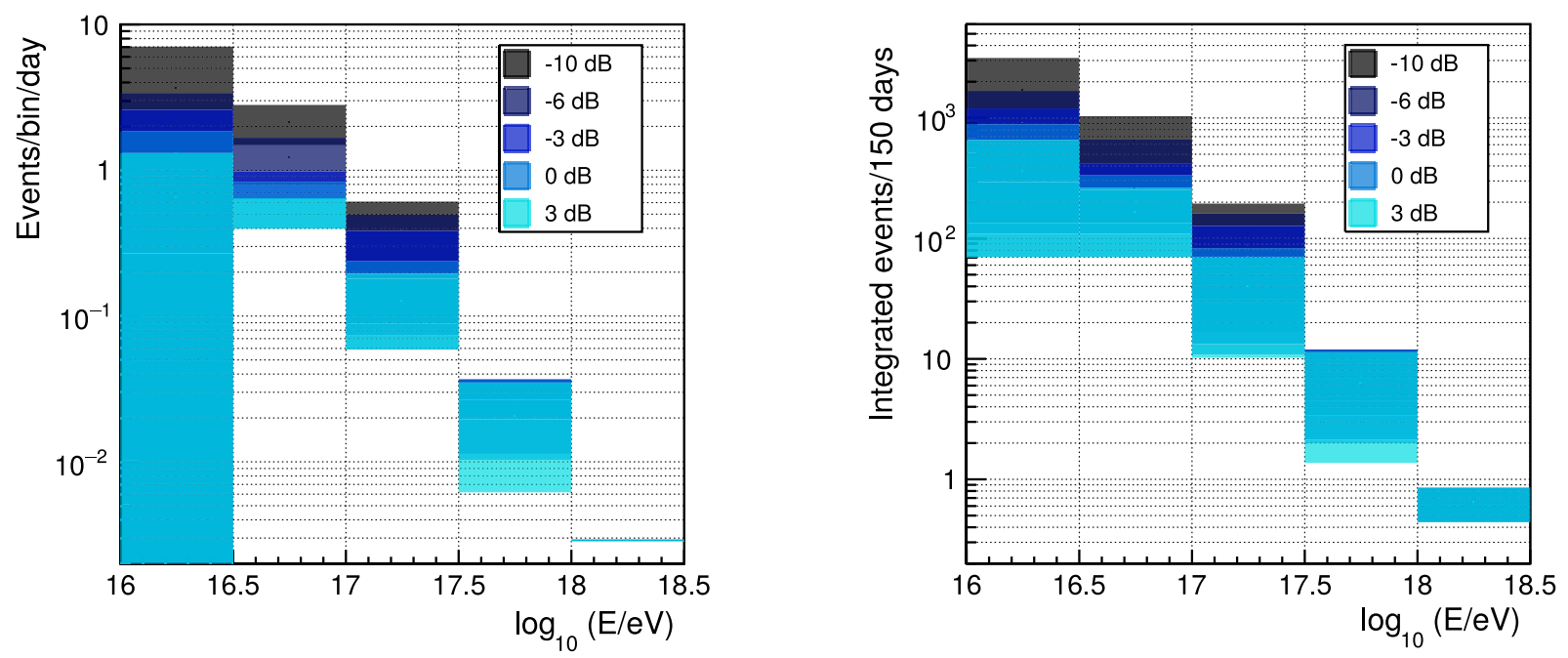

FIG. 7. (Left) Event rates per day as a function of energy for the RET-CR detector. Colors correspond to different signal-to-noise relative to thermal noise of $8 \mu \mathrm{V}$ for a $100 \mathrm{MHz}$ bandwidth. Width of the bands are explained in the text. (Right) Integrated event rates per 150 days as a function of energy for the RET-CR detector. Each bin represents the total integrated number of events per 150 days at and above that energy. Colors correspond to different signal-to-noise relative to thermal noise.

to cosmic rays from the full sky, $\int d \Omega=2 \pi$ sr; the aperture for RET-CR is from $0-30^{\circ}$ zenith, $\int d \Omega \approx 0.26 \pi \mathrm{sr}$.

We note that the measured flux of cosmic rays differs per experiment. A global study seeking to quantify this fluctuation between experiments [64] shows roughly $20 \%$ spread in measured energies between various experiments in our range of interest, leading to an uncertainty in the true flux. Therefore, to account for this uncertainty, we use the cosmic ray flux normalization in line with the mean of the global fit. A goal of the forthcoming CrossCalibration Array [33] is to mitigate this uncertainty between experiments.

Figure 7, left, presents our expected event rate per day as a function of energy for various signal-to-noise levels relative to a thermal noise rms of $8 \mu \mathrm{V}$. This is for a $160 \mathrm{~W}$ transmitter at $100 \mathrm{MHz}$ with a $10 \mathrm{~ns}$ plasma lifetime, a likely plasma lifetime for polar ice near the surface [24]. The upper and lower bounds of the bands correspond to the over and underestimated energy simulation respectively. The mean of the $0 \mathrm{~dB}$ signal to noise ratio (SNR) curve integrates to roughly 1 event per day.

Figure 7, right, shows the integrated event rate for one austral running season, approximately 150 days. An entry here represents the integrated number of events detected per 150 days at and above that energy, at the indicated SNR. We expect, e.g., $\sim 50$ events at and above $10^{17} \mathrm{eV}$ per season at the $0 \mathrm{~dB}$ threshold level.

For comparison to RET-N, the in-ice cascade energy for a $10^{17} \mathrm{eV}$ primary detected by RET-CR is roughly $10^{16} \mathrm{eV}$. This cascade energy corresponds to that of a chargedcurrent neutrino-nucleon interaction of $10^{16} \mathrm{eV}$ or a neutral current neutrino-nucleon interaction at $\sim 5 \times 10^{16} \mathrm{eV}$ for inelasticity $y \sim 0.2$. Thus, the primary cosmic ray energies probed with RET-CR are similar to those of neutrinos to be targeted with RET-N.

\section{CONCLUSION}

We have presented the Radar Echo Telescope for cosmic rays, a pathfinder in situ detector to test the radar echo method. Using the dense in-ice shower core of a cosmic ray air shower as a test beam, RET-CR will train trigger routines, energy and direction reconstruction methods, and analysis techniques to be employed by an eventual full-scale next-generation neutrino detector.

\section{SOFTWARE}

CORSIKA version 7.7400 (with QGSJETII-04 and URQMD 1.3cr), CORSIKA 7.7100 (with QGSJETII-04 and GHEISHA 2002d), CoREAS version 1.4 with a typical Taylor Dome, Antarctica atmosphere, GEANT4 versions 10.5 and 9.6, and RadioScatter version 1.1.0 were used to produce results for this paper.

\section{ACKNOWLEDGMENTS}

RET-CR is supported by the National Science Foundation under Grant Nos. NSF/PHY-2012980 and No. NSF/PHY-2012989. This work is also supported by the Flemish Foundation for Scientific Research FWO$12 Z$ D920N, the European Research Council under the European Unions (EU) Horizon 2020 research and innovation programme (Grant Agreement No. 805486), and the Belgian Funds for Scientific Research (FRS-FNRS). A.C. acknowledges support from NSF Grant No. 1806923. S. W. was supported by NSF CAREER 
Grant Nos. 1752922 and No. 2033500. D. B. is grateful for support from the U.S. National Science FoundationEPSCoR (RII Track-2 FEC, Grant ID 2019597). We express our gratitude to R. Dallier, L. Martin, J.-L. Beney, and the CODALEMA experiment for providing electronics and hardware to be used in the surface radio stations of RET-CR. Computing resources were provided by the Ohio Supercomputer Center.

\section{APPENDIX A: STATION LAYOUT}

The station layout for RET-CR is shown in Fig. 8. A phased transmitter is centrally located along with a data acquisition system and an amplifier enclosure for the transmitter. Three $1 \mathrm{~kW}$ solar power arrays are oriented in a triangle to maximize power over the full austral summer day. Satellite communications are shown near the solar power array. Each receive antenna is a vertical phased dipole array to maximize gain in an azimuthally symmetric region near the surface. The cosmic ray detector system is shown in blue, where each of the six, two-panel stations is shown in blue. The receive antennas are arranged in two sets, near at $20 \mathrm{~m}$ from the TX and as far as $100 \mathrm{~m}$ from the TX. The drawing is not to scale.

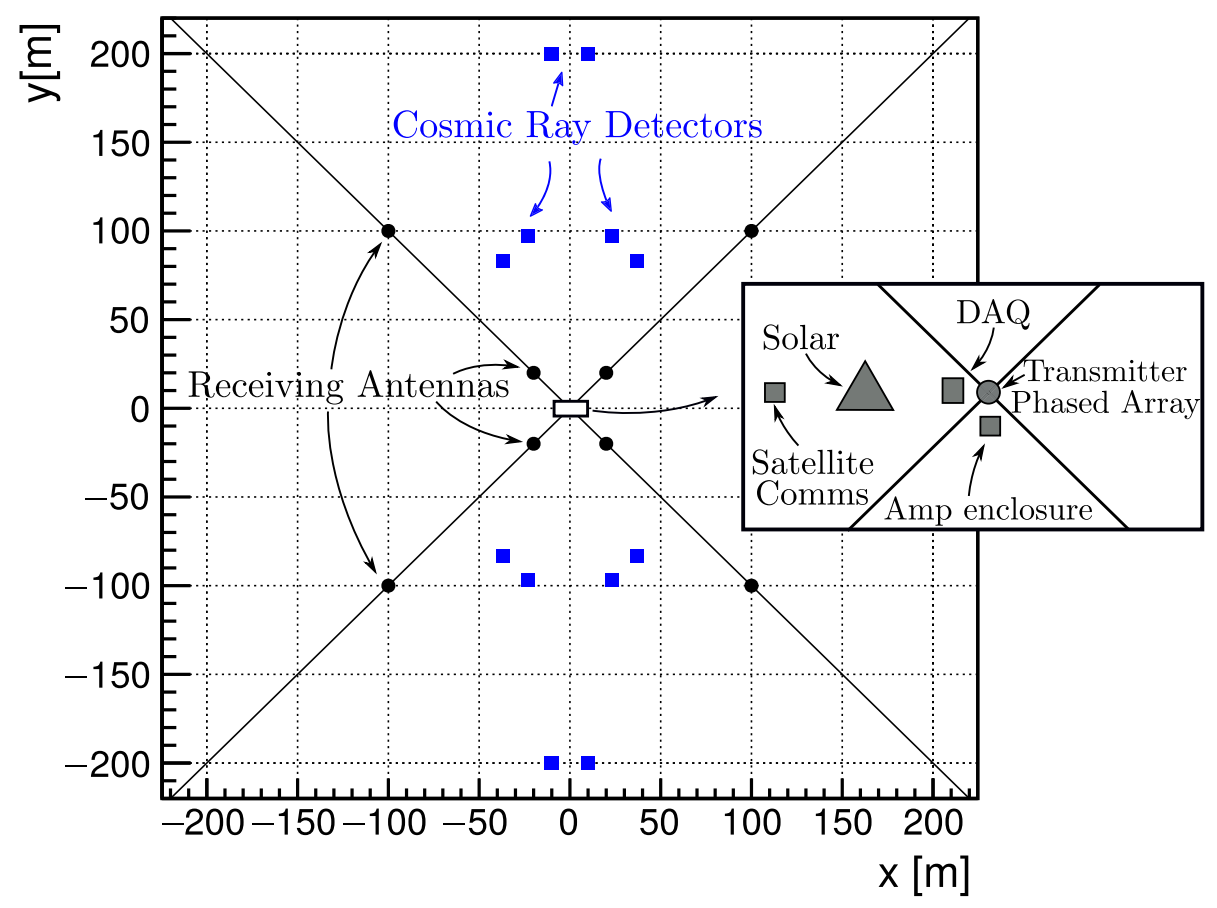

FIG. 8. The station layout for RET-CR. A phased transmitter array is centrally located along with a data acquisition system (DAQ) and an amplifier enclosure for the transmitter power amplifier(s). Three $1 \mathrm{~kW}$ solar power arrays oriented in a triangle are indicated along with satellite communications. The cosmic ray detector system is shown in blue. These serve to trigger the DAQ. The dimensions of the station are also indicated. 


\section{APPENDIX B: SYSTEM DIAGRAM}

A schematic of the RET-CR system, including the surface system, is shown in Fig. 9.

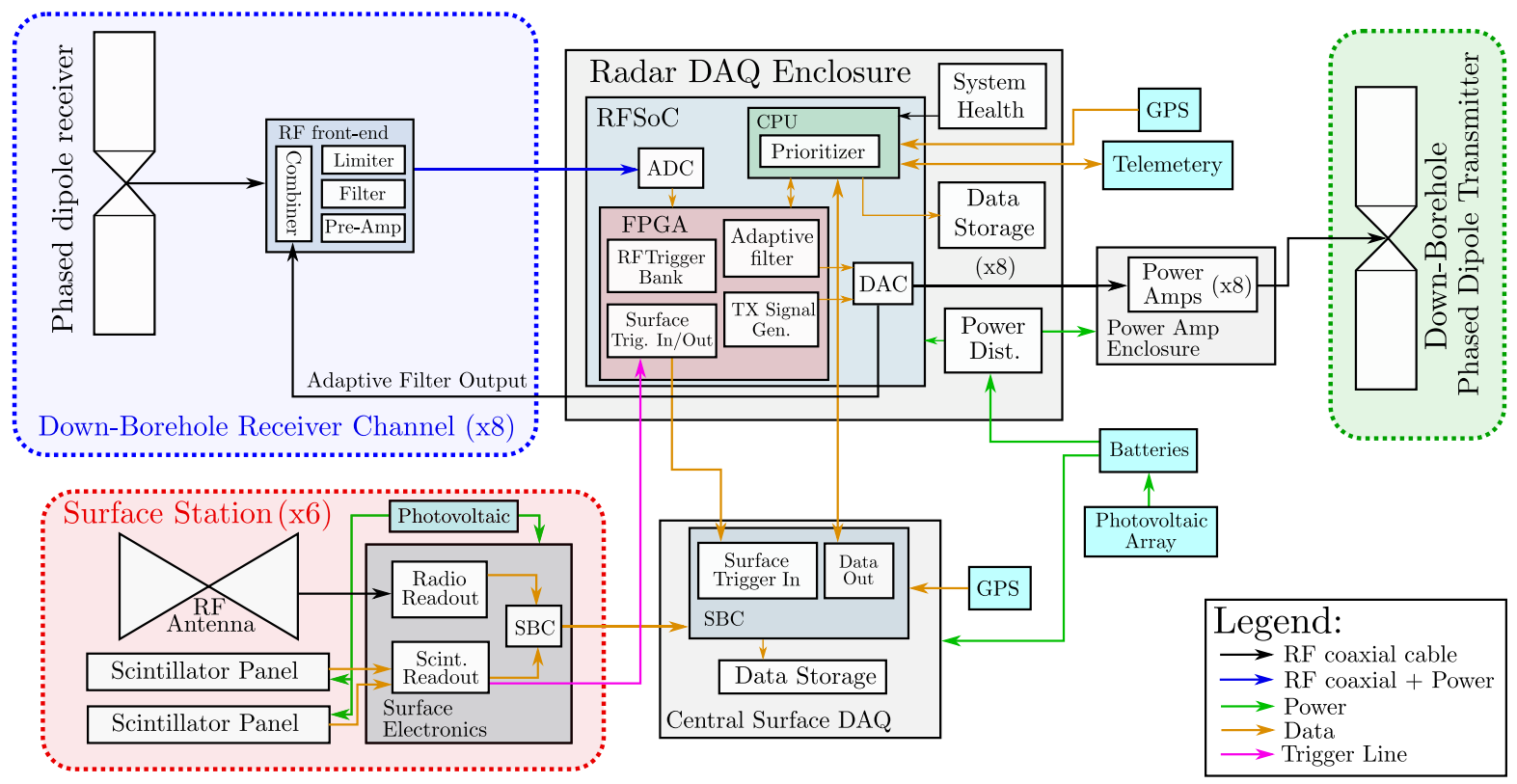

FIG. 9. The system diagram for RET-CR. The down-borehole receiver system is indicated on the left. There are eight identical receivers placed according to the layout shown in Fig. 8. The line indicating rf cable + power going to the receiver channel is powered via the bias tee. The down-borehole transmitter is an eight-channel phased array, each antenna having its own DAC channel and power amplifier. The surface system, shown on the bottom, has six identical individual stations (bottom left).

[1] M. Aartsen, M. Ackermann, J. Adams, J. A. Aguilar, M. Ahlers, M. Ahrens, I. Al Samarai, D. Altmann, K. Andeen et al., Science 361, eaat1378 (2018).

[2] B. P. Abbott, R. Abbott, T. D. Abbott, F. Acernese, K. Ackley, C. Adams, T. Adams, P. Addesso, R. X. Adhikari, V. B. Adya et al., Astrophys. J. 848, L12 (2017).

[3] M. Aartsen et al. (IceCube Collaboration) Science 361, 147 (2018).

[4] I. Kravchenko et al., Phys. Rev. D 73, 082002 (2006).

[5] P. Allison et al., Astropart. Phys. 35, 457 (2012).

[6] P. W. Gorham et al. (ANITA Collaboration), Astropart. Phys. 32, 10 (2009).

[7] S. R. Klein, IEEE Trans. Nucl. Sci. 60, 637 (2013).

[8] S. Wissel et al., J. Cosmol. Astropart. Phys. 11 (2020) 065.

[9] A. V. Olinto et al., Proc. Sci. ICRC2017 (2018) 542 [arXiv:1708.07599].

[10] J. Alvarez-Muñiz et al. (GRAND Collaboration), Sci. China Phys. Mech. Astron. 63, 219501 (2020).

[11] M. Chiba et al., arXiv:0710.4186v1.

[12] M. Chiba et al., AIP Conf. Proc. 1535, 45 (2013).

[13] K. D. de Vries, K. Hanson, and T. Meures, Astropart. Phys. 60, 25 (2015).
[14] S. Prohira and D. Besson, Nucl. Instrum. Methods Phys. Res., Sect. A 922, 161 (2019).

[15] S. Prohira et al., Phys. Rev. D 100, 072003 (2019).

[16] S. Prohira et al., Phys. Rev. Lett. 124, 091101 (2020).

[17] A. C. B. Lovell, Notes Records R. Soc. 47, 119 (1993).

[18] T. Matano, M. Nagano, K. Suga, and G. Tanahashi, Can. J. Phys. 46, S255 (1968).

[19] T. Terasawa, T. Nakamura, H. Sagawa, H. Miyamoto, H. Yoshida, and M. Fukushima, Proceedings of the 31st International Cosmic ray Conference (2009).

[20] R. Abbasi et al., Nucl. Instrum. Methods Phys. Res., Sect. A 767, 322 (2014).

[21] R. U. Abbasi et al., Astropart. Phys. 87, 1 (2017).

[22] J. Stasielak, R. Engel, S. Baur, P. Neunteufel, R. Šmída, F. Werner, and H. Wilczyński, Astropart. Phys. 73, 14 (2016).

[23] M. I. Bakunov, A. V. Maslov, A. L. Novokovskaya, and A. Kryemadhi, Astropart. Phys. 33, 335 (2010).

[24] M. P. De Haas, M. Kunst, J. M. Warman, and J. B. Verberne, J. Phys. Chem. 87, 4089 (1983).

[25] S. De Kockere, K. de Vries, and N. van Eijndhoven, Proc. Sci. ICRC2021 (2021) 1032. 
[26] K. Kamata and J. Nishimura, Prog. Theor. Phys. Suppl. 6, 93 (1958).

[27] K. Greisen, Annu. Rev. Nucl. Sci. 10, 63 (1960).

[28] D. Seckel, S. Seunarine, J. Clem, and A. Javaid, in Proceedings of the 30th International Cosmic Ray Conference, Merida, Mexico (2007).

[29] K. D. de Vries, S. Buitink, N. van Eijndhoven, T. Meures, A. Ó Murchadha, and O. Scholten, Astropart. Phys. 74, 96 (2016).

[30] D. Heck, J. Knapp, J. Capdevielle, G. Schatz, T. Thouw et al., Rep. Fzka 6019 (1998).

[31] S. Agostineli et al., Nucl. Instrum. Methods Phys. Res., Sect. A 506, 250 (2003).

[32] S. Prohira, https://github.com/prchyr/RadioScatter (2017).

[33] K. Mulrey, Proc. Sci. ICRC2021 (2021) 414.

[34] K. Mulrey et al., J. Cosmol. Astropart. Phys. 11 (2020) 017.

[35] H. Bozdog et al., Nucl. Instrum. Methods Phys. Res., Sect. A 465, 455 (2001).

[36] B. Revenu, D. Charrier, R. Dallier, A. Escudie, D. GarcíaFernández, A. Lecacheux, and L. Martin, Proc. Sci. ICRC2017 (2018) 416.

[37] D. Ardouin, A. Belletoile, D. Charrier, R. Dallier, L. Denis, P. Eschstruth, T. Gousset, F. Haddad, J. Lamblin, P. Lautridou et al., Astropart. Phys. 26, 341 (2006).

[38] S. Thoudam et al., Nucl. Instrum. Methods Phys. Res., Sect. A 767, 339 (2014).

[39] T. Huege, M. Ludwig, and C. W. James, AIP Conf. Proc. 1535, 128 (2013).

[40] A. Corstanje et al., Astropart. Phys. 61, 22 (2015).

[41] W. D. Apel et al. (LOPES Collaboration), Eur. Phys. J. C 81, 176 (2021).

[42] B. Revenu, D. Charrier, R. Dallier, A. Escudie, D. GarcíaFernández, A. Lecacheux, and L. Martin, J. Phys. Conf. Ser. 1181, 012029 (2019).

[43] A. Aab et al. (Pierre Auger Collaboration), J. Instrum. 11, P01018 (2016).

[44] D. Kostunin, P. A. Bezyazeekov, R. Hiller, F. G. Schröder, V. Lenok, and E. Levinson, Astropart. Phys. 74, 79 (2016).

[45] A. Aab et al. (Pierre Auger Collaboration), Phys. Rev. D 93, 122005 (2016).
[46] C. Welling, C. Glaser, and A. Nelles, J. Cosmol. Astropart. Phys. 10 (2019) 075.

[47] Xilinx, https://www.xilinx.com/products/silicon-devices/ soc/rfsoc.html (2021).

[48] S. Kunwar et al., Nucl. Instrum. Methods Phys. Res., Sect. A 797, 110 (2015).

[49] S. Prohira, S. Kunwar, K. Ratzlaff, R. Young, and D. Besson, Nucl. Instrum. Methods Phys. Res., Sect. A 890, 126 (2018).

[50] P. Allison et al., Nucl. Instrum. Methods Phys. Res., Sect. A 930, 112 (2019).

[51] A. F. Oskooi, D. Roundy, M. Ibanescu, P. Bermel, J. D. Joannopoulos, and S. G. Johnson, Comput. Phys. Commun. 181, 687 (2010).

[52] S. Prohira et al. (Radar Echo Telescope Collaboration), Phys. Rev. D 103, 103007 (2021).

[53] S. Prohira, https://github.com/prchyr/paraPropPython (2020).

[54] J. C. Hanson, Electronics 10, 415 (2021).

[55] A. Anker et al., J. Cosmol. Astropart. Phys. 11 (2019) 030.

[56] P. Zyla et al. (Particle Data Group Collaboration), Prog. Theor. Exp. Phys. 2020, 083C01 (2020).

[57] K. C. Kuivinen, Antarct. J. U.S. 18, 113 (1983).

[58] R. L. Hawley, E. M. Morris, and J. R. McConnell, J. Glaciol. 54, 839 (2008).

[59] R. J. Arthern, H. F. J. Corr, F. Gillet-Chaulet, R. L. Hawley, and E. M. Morris, J. Geophys. Res. 118, 1257 (2013).

[60] G. d. Q. Robin, J. Glaciol. 15, 151 (1975).

[61] C. Deaconu, A. G. Vieregg, S. A. Wissel, J. Bowen, S. Chipman, A. Gupta, C. Miki, R. J. Nichol, and D. Saltzberg, Phys. Rev. D 98, 043010 (2018).

[62] M. G. Aartsen et al. (IceCube Collaboration), Phys. Rev. D 88, 042004 (2013).

[63] A. Aab et al. (Pierre Auger Collaboration), Phys. Rev. Lett. 125, 121106 (2020).

[64] H. P. Dembinski, R. Engel, A. Fedynitch, T. Gaisser, F. Riehn, and T. Stanev, Proc. Sci. ICRC2017 (2018) 533 [arXiv:1711.11432]. 